\title{
Split delivery scheme for vehicle supported UAV
}

\author{
Luying Dong, Shuhan Ma, Yajuan Han, and Yipeng Zhou* \\ School of E-commerce and Logistics, Beijing Technology and Business University, Beijing, 100048, \\ China
}

\begin{abstract}
The traditional demand split logistics distribution schemes are mainly based on vehicle distribution. In recent years, with the rapid development of civil UAVs, the use of UAVs for distribution will be more efficient and economical in some specific conditions. In this paper, through the analysis of the advantages of UAV distribution, we simulate the demands and distribution schemes in real scene, especially in remote mountainous areas. And then, we propose to define a demand-splitting distribution scheme of vehicle-supported UAV, which ensures the customer point will be satisfy demand when the loading capacity of the UAV is exceeded. This scheme aims to provides a realizable distribution scheme for the customers point in mountainous areas with large demand.
\end{abstract}

Keywords: Vehicle supported UAV distribution, Split delivery, Terminal distribution.

\section{Introduction}

In recent years, the logistics service industry has developed rapidly, and the business volume has repeatedly hit new highs. People's requirements on the coverage degree and distribution efficiency of logistics have become increasingly high. Nonetheless, the problem of difficult and expensive distribution in remote areas has not been solved yet. With the development of science and technology, UAV technology is becoming more and more mature. Using UAV for distribution can not only save time and costs, but also send goods to remote areas such as mountainous areas, which further expands the range of distribution. However, The payload of the present UAVs used for delivery is less than 10 $\mathrm{kg}$, most of which are 1 to $2 \mathrm{~kg}$. When the demand for a customer site outgrows the maximum loading capacity of the UAVs used, the demand for that customer site cannot be met[1]. When conducting distribution according to the existing civil unmanned aerial vehicles (UAVs), in this case, it is necessary to split the needs of customer points and assign them multiple UAVs for distribution. Based on this, this paper proposes a demandable distribution scheme of vehicle support for UAVs, which we believe is conducive to better solving the distribution problems in remote mountainous areas.

\footnotetext{
*Corresponding author: yipengzhou@163.com
} 


\section{Current situation of UAV distribution}

As the "black technology" of logistics distribution, UAV is a sharp tool to solve the problem of "last mile" distribution efficiency[2-3]. Compared with human distribution, UAV has the advantage of being intelligent, informatized, and unmanned, and has higher distribution efficiency, which is deeply concerned by express delivery, e-commerce, and other industries.

\subsection{The current application scenarios of UAV distribution are as follows:}

Distribution of medical supplies: On February 6, 2020, Antwork built the first anti-epidemic "urban air transport channel" in China through UAV + unmanned station[4].

Distribution of living materials: With the support of Hebei Baiyangdian government, Jingdong Logistics UAV team established air transportation routes to distribute living materials for villagers through environmental survey and airspace approval.

\subsection{Advantages of UAV distribution:}

Fast delivery:Today, consumers are constantly putting forward new requirements for delivery timeliness. "Two-hour delivery" and "instant delivery" have gradually become the new voice of consumers. UAVs delivery can realize the urgent business of intracity logistics. It is free from traffic jams, waiting for red lights, couriers to find the address and other problems, as it can instantly find a short-cut with the support of digital maps, greatly shortening the delivery time[5].

Low delivery cost:In China, rural online shopping users live scatteredly, the road is not smooth,the traffic is inconvenient, and the distribution volume is small, which leads to long distribution routes and high distribution costs. According to statistics, the cost of rural delivery is five times that of urban delivery. UAVs have the advantages of convenient deployment, low cost, strong mobility, and low requirements for the task environment. Using UAVs to replace the traditional means of transportation for express delivery can better solve the problem of express delivery cost[6].

Low requirements for delivery conditions:In some poor mountain villages of our country, the natural environment is bad with poor road conditions, and the traditional delivery vehicles can not reach, so they can only be reached by walking. At the same time, the exhibition center, shopping malls, shopping malls, and other densely populated places in the city are inaccessible by vehicles. UAV has the advantages of convenient deployment, low cost, and strong mobility, and will not be limited by complex terrain. UAV distribution can better solve the above problems [7-8].

\section{Demand of vehicle supporting UAV can be split and distributed}

In 1989, Dror and Trudeau first proposed the vehicle routing problem with split delivery, which means that a group of different vehicles start from the yard and visit each customer point with known demand. In one service, each customer point may not be visited by one vehicle only once, that is, multiple deliveries can jointly meet the demand of a customer point. Because of the limit that a single customer point can only be visited once, the number of vehicles can be reduced to the greatest extent and the distribution cost can be reduced.

With the rapid development of civil UAVs, using UAVs for "last mile" distribution is the best choice to save manpower, material resources, time, and costs. However, the loading capacity of UAV is limited. If the demand exceeds the maximum loading capacity of a single $\mathrm{UAV}$, the demand of the customer point cannot be met without splitting the demand, or 
multiple deliveries. At this time, to meet customer needs and save time, it is necessary to split the demand of the customer point. $1[9]$.

The schema chart of split delivery scheme for vehicle supporting UAV is shown in Figure

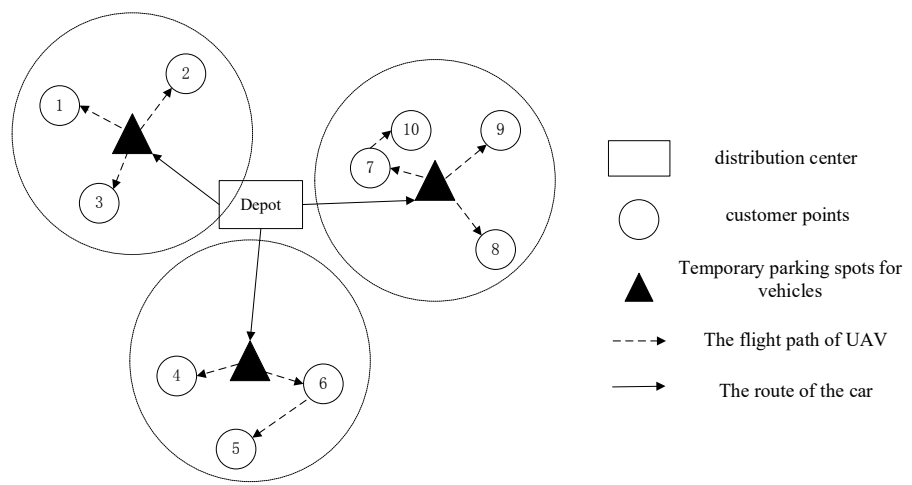

Fig. 1. Schema chart of split delivery scheme for vehicle supporting UAV.

\section{Scheme description}

The distribution problem of vehicle-supported UAV is a three-level network, which consists of a distribution center, T vehicles, R UAVs, and K customer points. After obtaining the demand information from multiple distribution points, the distribution center arranges vehicles and UAVs for distribution. Vehicles carrying UAVs from the distribution center, according to a certain path to choose a temporary stop. The models of UAVs carried on the vehicles are the same, and the maximum loading capacity is fixed and known. For the customer point whose demand is greater than the maximum loading capacity of UAV, it is necessary to split the demand and distribute it by different UAVs; that is, the customer point will be visited by UAVs many times. After the UAVs complete the distribution, they return to the vehicle empty, and then the vehicles are brought back to the distribution center to start the next distribution. In order to save time and cost, we need to use the least number of UAVs and the shortest distance.

The structure diagram of the split delivery scheme for vehicle-supported UAV is shown in Figure 2.

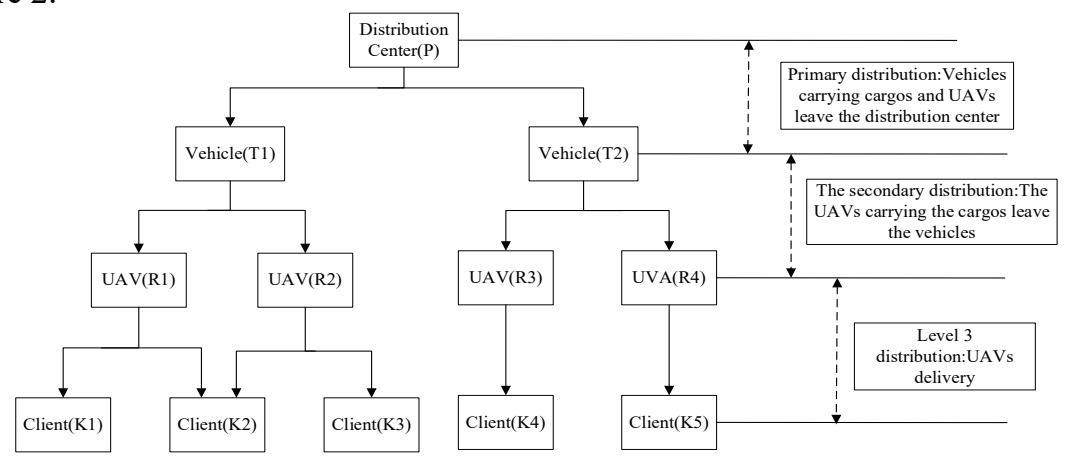

Fig. 2. Structure diagram of split delivery scheme for vehicle supporting UAV.

The flow chart of split delivery scheme for vehicle supported UAV is shown in Figure 3. 


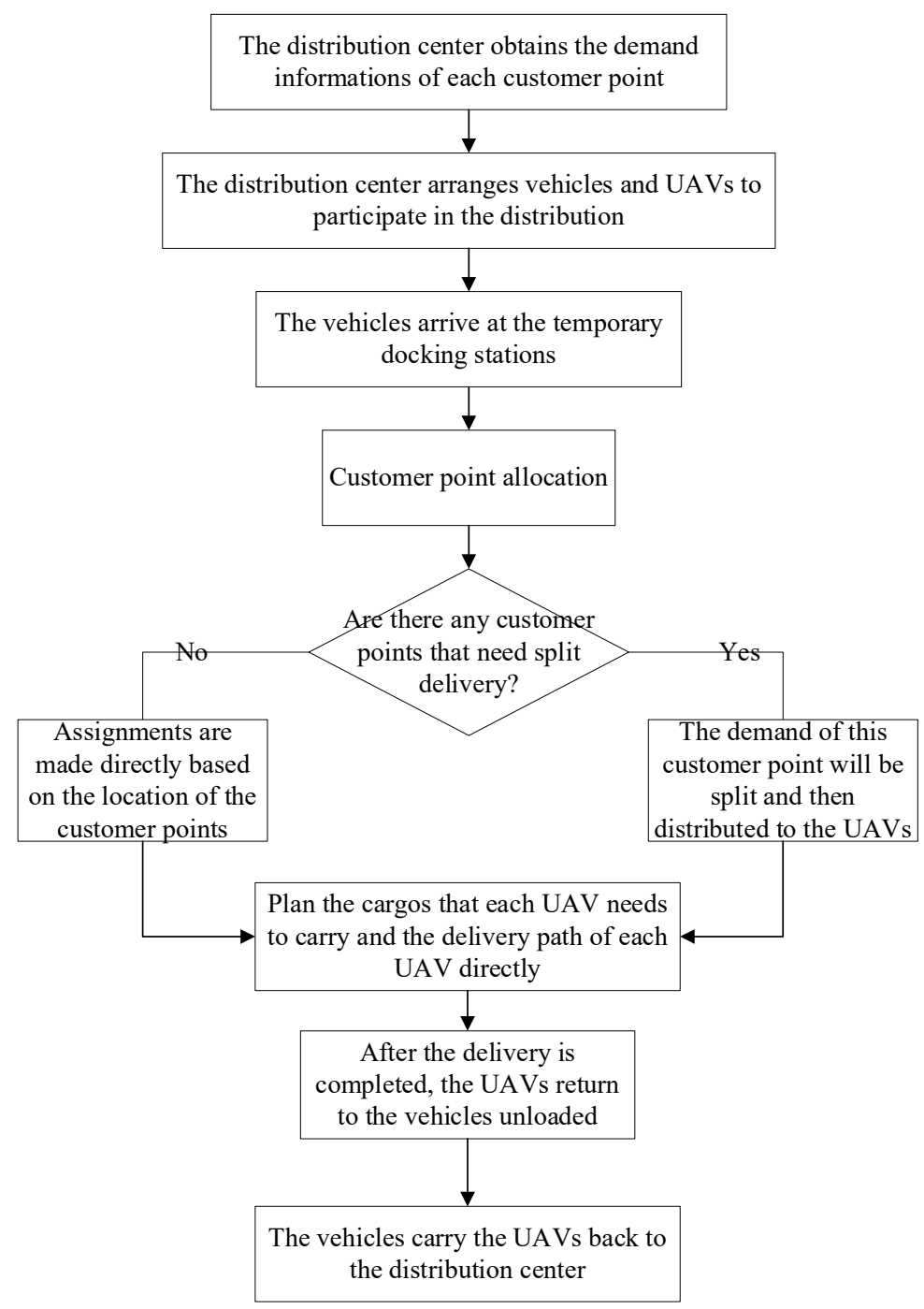

Fig. 3. Flow chart of split delivery scheme for vehicle supporting UAV.

\section{Scene description}

Due to the remote location, the lack of a perfect road traffic system in remote areas and mountainous areas, it is difficult to use vehicles for logistics distribution, or even to carry out the distribution. In these areas, the population density is often low, and the cost of logistics distribution is very high. As a consequence of the rapid popularity of online shopping in recent years, the demand for distribution in remote areas is increasing, so it is urgent to reduce the cost of distribution to these areas. The UAV designed for express delivery is equipped with the most advanced navigation system, which can quickly deliver the package to the designated place according to the present route. Moreover, for distribution to remote areas, UAVs also have great advantages in costs[10]. However, if the demand of a certain customer point is too large for a single UAV delivery to meet the demand of the customer point, it is necessary to split the demand of the customer point, and use multiple UAVs to visit the customer point many times to meet the distribution demand. 


\section{Conclusion}

In this paper, a solution is proposed to solve the problem that the large demand customer point can not be met, which is to split the demand of large demand customer point and then to distribute by using UAVs. The scheme is aimed to promote the development of express delivery in mountainous areas. In the era of expanding logistics market demand, the logistics demand in remote areas can not be underestimated. Considering this situation, this paper puts forward the scheme to solve the problems of difficulty and the high cost of distribution to remote areas. But the specific implementation of the scheme also needs the support of the algorithm, such as the path planning of the vehicles, the flight path planning of the UAVs using the SDVRP model, and the time window constraint. The future research of the scheme can be carried out in combination with the above three points.

\section{References}

1. Yang Shuangpeng, Guo Xiuping, Gao Jiaojiao. Research on the Joint Delivery Problem of "Non-contact" Truck + UAV [J/OL].Industrial engineering and management: 1-17 [2021-05-19].

2. Aggarwal S, Kumar N. Path planning techniques for unmanned aerial vehicles: A review, solutions, and challenges[J]. Computer Communications, 2020, 149(10): 270-299.

3. Chung S H, Sah B, Lee J. Optimization for drone and drone-truck combined operations: A review of the state of the art and future directions [J]. Computers and Operations Research, 2020, 123(5): 1-29.

4. Zhang Wenbin, $\mathrm{Wu}$ Leiming, Gao Bo.Practice and enlightenment of unmanned delivery for COVID-19 epidemic prevention and control [J]. Journal of Military Transportation University, 2020, 22(05):57-60.

5. Cao Xinhui. Community Intelligent UAV Delivery System Based on Machine Vision [J]. Communications World, 2019, 26(2):259-261.

6. Ren Xinhui, Wang Liu, Zou Xintong.Research on the innovation mode of urban instant delivery based on multi-factors [J]. Commercial Economics Research,2020(11):133-136.

7. Bao Xinxin, Luo Pei, Wang Kelin. Advantages and obstacles of UAV distribution [J]. Modern commerce, 2017 (23): 13-14

8. Han Ming, Wang Yabin, Ding Lianyong, Wang Tianxing.Optimization of Vehicle + UAV Distribution Path Based on CTDEA Algorithm [J]. Journal of Ordnance Equipment Engineering,2019, 40(11):149-154.

9. Yang shuangpeng, Guo Xiuping, Gao Jiaojiao. Research on joint distribution of "contactless" truck and UAV [J / OL]. Industrial engineering and management: 1-17 [2021-05-12] http://kns.cnki.net/kcm s/detail/31.1 738.t.20210330.1002.002.html.

10. Liu Xingchen, Zhang Mei. Research on the design of logistics distribution UAV and its distribution system [J]. Science and technology innovation guide. 2017,14 (21) http://kns.cnki.net /kcms/detail/31.1738.t.20210330.1002.002.html. 